\title{
Assessment of EEG Changes in Neonatal Sepsis at Al-Zahraa University Hospital's NIC Unit
}

\author{
Sara Tarek Mohamed Mohamed ${ }^{1 *}$, Zeinab Farag Oshaiba², Marwa El-Hady Abd El Moneim², \\ Asmaa Abd El Wakeel Ibrahim² \\ ${ }^{1}$ Student Hospital, Al-Azhar University, Cairo, Egypt \\ ${ }^{2}$ Pediatric and Neonataology Department of Al Zahraa University Hospital, Faculty of Medicine for Girls, Al-Azhar University, \\ Cairo, Egypt \\ Email:^drsarata-rek1991@gmail.com,dr_z.farag@yahoo.com,marwaelhady93@yahoo.com, asmaawakeel@yahoo.com
}

How to cite this paper: Mohamed, S.T.M., Oshaiba, Z.F., El Moneim, M.E.-H.A. and Ibrahim, A.A.E.W. (2020) Assessment of EEG Changes in Neonatal Sepsis at Al-Zahraa University Hospital's NIC Unit. Open Journal of Pediatrics, 10, 493-503.

https://doi.org/10.4236/ojped.2020.103050

Received: July 15, 2020

Accepted: September 8, 2020

Published: September 11, 2020

Copyright $\odot 2020$ by author(s) and Scientific Research Publishing Inc. This work is licensed under the Creative Commons Attribution International License (CC BY 4.0).

http://creativecommons.org/licenses/by/4.0/

\begin{abstract}
Background: EEG is one of the most sensitive instrumental techniques for identifying sepsis-related neurological complications and a valuable tool in the ICU when clinical assessment is difficult. Aim of the Study: To detect EEG changes in neonates with evidence of sepsis. Patients and Methods: This was a prospective observational study for detecting EEG changes in cases of proven neonatal sepsis. This study was conducted at the NICU of Al Zahraa University Hospital for a period from October 2019 to March 2020. Our studied neonates were 50. This study included full-term newborns (37 to 42 weeks of gestational age) with clinical and laboratory evidence of sepsis (early and late onset). Neonatal birth asphyxia, jaundice, congenital malformations, suspected inborn error of metabolism and neonates electrolytes disturbances were excluded from the study. We record EEG changes during 20 min By an electroencephalogram (EEG) with abioelectric amplifier (Deltamed, Bourgogne), (Philips) Screen. Results: Among our studied neonates (neonates with evidence sepsis), $44.0 \%$ developed clinical seizures, $81 \%$ of the seizures group had abnormal EEG, 35.7\% of No seizures group had abnormal EEG. Among our studied neonates, 56\% had abnormal EEG EEG abnormalities were as follow, $26 \%$ / had low voltage, $30 \%$ had spikes, $22 \%$ had sharp waves. Conclusion: About 35\% (one third) of neonates who didn't develop clinical seizures had abnormal EEG, suggesting a poor correlation between clinical and electroencephalographic detection of seizures.
\end{abstract}

\section{Keywords}

Background Abnormalities, Electroencephalogram, Neurological Complication, Sepsis Spike, Seizures 


\section{Introduction}

Neonatal bacteremia remains one of the major infectious problems in the neonatal intensive care unit (NICU) with mortality rates of $10 \%-30 \%$. It is also associated with increased medical costs, prolonged hospital stay and potentially poor long-term neurodevelopmental outcomes [1] (Yismaw et al., 2019).

Neurological complications due to sepsis may result from either direct bacterial invasion, sepsis-associated encephalopathy [2] (Katie, 2019).

Neurological complications after neonatal bacteremia with or without meningitis are important, because they can be life-threatening and may require neurosurgical treatment. There are a large number of studies on the incidence, risk factors, microbiology, and mortality of neonatal bacteremia or meningitis. Moreover, an episode of bacteremia presenting with severe sepsis or septic shock may be associated with neurological sequelae, probably due to brain hypoperfusion, transient hypoxia, or metabolic acidosis [3] (Paczosa and Mecsas, 2016).

EEG is one of the most sensitive instrumental techniques for identifying sepsis-related neurological complications and a valuable tool in the ICU when clinical assessment is difficult [4] (Molnar et al., 2018).

The presence and severity of EEG background abnormalities are associated with altered clinical, laboratory, and neurologic findings, as well as the severity of septic neurological insult and increased mortality [5] (Gilmore et al., 2015).

Seizures are the most common and the most frequent neurological presentation in neonatal sepsis. They are a major risk of death and long-term morbidity including neuro developmental disabilities [6] (Talebian et al., 2015).

Seizures constitute the most frequent and distinctive manifestation of neurological disturbance in the neonatal period and have the highest incidence in the first month of life. They are a major risk for death or subsequent neurological disability and can independently confer an adverse neuro developmental outcome in high-risk neonates. It is important to determine the underlying etiology of neonatal seizures in order to effectively treat the underlying cause and to offer a reasonably accurate prognosis to parents [7] (Grova et al., 2019).

A common recording method for diagnostic purposes is electroencephalography (EEG), where electrodes are placed on the surface of the scalp for recording the average electrical activity from different areas of the cortex [8] ( $\mathrm{Ra}$ mantani et al., 2016).

\section{Aim of the Study}

This is a prospective observational study was done at NICU of Al-Zahraa University Hospital; aiming to detect EEG changes in cases of neonates with evidence of sepsis.

\section{Patients and Methods}

This was a prospective observational study for detecting EEG changes in cases of proven neonatal sepsis. This study was conducted at the NICU of Al Zahraa 
University Hospital on 50 neonates with sepsis for a period from October 2019 to March 2020. The procedures and the aim of the study were explained to the child's care giver. The neonates were 26 males and 24 females. This study included full-term newborns ( 37 to 42 weeks of gestational age) and neonates with clinical and laboratory evidence of sepsis (early and late onset). Neonates with birth asphyxia, neonatal jaundice (kernicterus), congenital malformations, neonates with suspected inborn error of metabolism and neonates with electrolytes disturbances were excluded from the study.

The studied group was subjected to the following: Evidance neonatal sepsis clinically and laboratory. Full history taking including full detailed medical history especially antieplieptics drugs or sedative. Full laboratory investigations: $\mathrm{cbc}, \mathrm{c}$-reactive prtptine, blood culture, serum calcium and phosphorous, liver and kidney function test and serum blood glucose. Radiological examination through: using an electroencephalogram (EEG) with abioelectric amplifier (Deltamed, Bourgogne), (Philips) Screen. This study was conducted at the NICU of $\mathrm{Al}$ Zahraa University, time of recording $20 \mathrm{~min}$.

The impedance was kept below $5 \mathrm{k} \Omega$ during the recording. Observed clinical seizures and handling of the infant were recorded by the nursing staff. Electrical activity was detected by electrodes applied through head cap according to $10-20$ system for recording of EEG.

We use electrode cap for neonates. Electrode caps are color coded and can be adjusted to fit different head sizes. The neoinatal montage includes eight scalp electrodes (FP2, C4, T4, O2, FP1, C3, T3, O1) and additional three electrodes $(\mathrm{Fz}, \mathrm{Cz}, \mathrm{Pz})$ and The low-frequency filter is set lower in neonatal recordings at 0.005 to 0.01 or $0.5 \mathrm{~Hz}$.

The name of electrode sites use alphabetical abbreviations that identify the lobe or area of the brain which each electrode recordes from $\mathrm{F}=$ frontal $\mathrm{Fp}=$ frontopolar $\mathrm{T}=$ temoral $\mathrm{C}=\operatorname{central} \mathrm{P}=$ parietal $\mathrm{O}=$ occipital $\mathrm{A}=$ auricular (ear electrode). Even numbers identify electrode positions on the right side of head, and odd numbers refer to the left side. The label $(Z)$ points to electrode sites in the midline of the head. For example, $\mathrm{Cz}$ refers to the midline central region of the head. We use bipolar montages.

We take full term 50 neonates with sepsis (early and late onset sepsis) guided clinically and confirmed by blood culture.

Our studied neonates are categorized into two groups according to the presence of clinical seizures or not (sepsis, then we do EEG, time of recording 20 min, make sure that the neonates don't have any sedatives. We compare the seizures group and no seizures group. The types of seizures clinically were Motor (tonic and clonic) and subtle (subtle in the form of yawing, non-nutritive suckling, abnormal eye movement) and electroencephalographic changes like multiple spikes, multiple spike, abnormal background, sharp waves.

Limitation of our study: we use conventional not amplitude and low number of cases. 


\section{Statistical Analysis}

All data were subjected to revision and validation then description and analysis on IBM-compatible PC by using SPSS (Statistical Package for the Social Science) program version 22.0.0, Microsoft Office Excel 2007, and Graph Pad Prism 6.

Descriptive statistics were performed for all studied parameters in the three studied groups and were presented in the form of mean, median, standard deviation (SD), minimum, maximum, range, and percentages.

Analytical comparison between different groups was done by using student $t$-test and analysis of variance (ANOVA) for comparing parametric data when normally distributed. For comparing non-parametric data, Fisher's exact test was used instead of chi-square test as Fisher's test calculates an exact P-value, while chi-square only calculates an approximation.

The level of significance was calculated according to the following probability (P) values:

- $\mathrm{P}>0.05=$ non significant (NS);

- $\mathrm{P}<0.05=$ significant $(\mathrm{S})$;

- $\mathrm{P}<0.001=$ highly significant (HS).

\section{Results}

We had 50 neonates with evidence sepsis with included criteria. Among our studied neonates $52.0 \%$ were males and $48.0 \%$ were females, their mean gestational age was $37.94 \pm 1.35$. 86.0\% were delivered with Lscs. The most common maternal risk factors for sepsis were maternal DM and PROM $>18 \mathrm{~h}$. The commonest organism in blood culture was Klebsiella with $50.0 \%$ followed by with $30.0 \%$ Escherichia coli then Staphylococcus with $20.0 \%$. The clinical and laboratory data of included neonates were demonstrated in Table 1.

Among our studied neonates, $44.0 \%$ developed clinical seizures. The most common presentation of neonatal seizures was subtle seizures with $81 \%$ followed by tonic with $41.2 \%$ then clonic with $35.3 \%$, as demonstrated in Table 2 .

Among our studied neonates, 56\% had abnormal EEG. EEG abnormalities were as follow: $26 \%$ / had low voltage, $30 \%$ had spikes, $22 \%$ had sharp waves. Regarding the site of EEG abnormalities, $24 \%$ were centropartial and $20 \%$ were fronto temporal, regarding the side of EEG abnormality, 30\% were right sided and $26 \%$ were left sided, Table 3.

On comparing the EEG changes between neonates with and without clinical Seizures, in neonates with clinical seizures $18.2 \%$ had no EEG abnormalities and 81.2\% had abnormal EEG. In neonates with NO clinical seizures $35.7 \%$ had EEG abnormalities and $64.3 \%$ had normal EEG. Background abnormalities were detected in $40.9 \%$ of the neonates with seizures and $14.3 \%$ of the neonates without seizures, spikes were detected in $40.9 \%$ of the neonates with seizures and $21.4 \%$ of the neonates without seizures. In the seizures group 40.9\% EEG changes were left side, in the NO seizure group $21.4 \%$ of EEG changes were right sided. So there was highly statistically significant difference found between two groups 
regarding EEG changes, so we have poor correlation between clinical and electroencephalographic seizures (Table 4).

Table 1. Clinical and laboratory data of studied neonates.

\begin{tabular}{|c|c|c|}
\hline & & No. $=50$ \\
\hline \multirow{2}{*}{ Sex } & Female & $24(48.0 \%)$ \\
\hline & Male & $26(52.0 \%)$ \\
\hline \multirow{2}{*}{ Gestational age (weeks) } & Mean \pm SD & $37.94 \pm 1.35$ \\
\hline & Range & $37-42$ \\
\hline \multirow{2}{*}{ Mode of delivery } & Lcs & $43(86.0 \%)$ \\
\hline & Spontaneous vaginal delivery & $7(14.0 \%)$ \\
\hline \multirow{5}{*}{ Maternal risk factor } & Chorioaminitis & $2(4.0 \%)$ \\
\hline & Diabetes mellitus & $8(16.0 \%)$ \\
\hline & No risk factors & $30(60.0 \%)$ \\
\hline & $\mathrm{PROM}>18 \mathrm{~h}$ & $6(12.0 \%)$ \\
\hline & Urinary tract infection & $4(8.0 \%)$ \\
\hline \multirow{3}{*}{ Blood. Culture } & Escherichia coli & $15(30.0 \%)$ \\
\hline & Klebsiella & $25(50.0 \%)$ \\
\hline & Staphylococcus & $10(20.0 \%)$ \\
\hline \multirow{2}{*}{$\mathrm{TLC}\left(\times 1000 / \mathrm{mm}^{3}\right)$} & Mean \pm SD & $15.02 \pm 4.3$ \\
\hline & Range & $8.8-25$ \\
\hline \multirow{2}{*}{ I/M Ratio } & Mean \pm SD & $0.25 \pm 0.08$ \\
\hline & Range & $0.06-0.39$ \\
\hline \multirow{2}{*}{ I/T Ratio } & Mean \pm SD & $0.25 \pm 0.07$ \\
\hline & Range & $0.12-0.36$ \\
\hline \multirow{2}{*}{ Hematological sepsis score } & Mean \pm SD & $3.22 \pm 1.53$ \\
\hline & Range & $1-6$ \\
\hline
\end{tabular}

Table 2. The frequency and type of seizures among the studied neonates.

\begin{tabular}{|c|c|c|}
\hline \multicolumn{2}{|c|}{ Variable } & \multirow{2}{*}{$\begin{array}{l}\text { No. }=50 \\
22(44.0 \%)\end{array}$} \\
\hline & Yes & \\
\hline Seizure & No & $28(56.0 \%)$ \\
\hline \multirow{5}{*}{ Type of Seizure } & Tonic & $7(41.2 \%)$ \\
\hline & Subtle & $18(81 \%)$ \\
\hline & Myoclonic & $2(11.8 \%)$ \\
\hline & Clonic & $6(35.3 \%)$ \\
\hline & Multifocal colonic & $2(11.8 \%)$ \\
\hline
\end{tabular}


Table 3. EEG changes among our studied neonates.

\begin{tabular}{cccc}
\hline \multirow{2}{*}{ EEG changes } & No. & $\%$ \\
\hline \multirow{2}{*}{ Background } & Normal & 22 & $44 \%$ \\
& Abnormal & 28 & $56 \%$ \\
\hline \multirow{3}{*}{ Discharges } & Low voltage & 13 & $26.0 \%$ \\
& Normal & 37 & $74.0 \%$ \\
\hline \multirow{2}{*}{ Site } & Polyspikes & 22 & $44.0 \%$ \\
& Sharp waves & 2 & $4.0 \%$ \\
& Spikes & 11 & $22.0 \%$ \\
& Centroparital & 15 & $30.0 \%$ \\
& Frontotempotal & 12 & $24.0 \%$ \\
& Multifocal & 10 & $20.0 \%$ \\
& No & 6 & $12.0 \%$ \\
Side & Left side & 22 & $44.0 \%$ \\
\hline & No & 13 & $26.0 \%$ \\
& Right side & 22 & $44.0 \%$ \\
& & 15 & $30.0 \%$ \\
\hline
\end{tabular}

Table 4. Comparison of EEG findings between neonates with and without seizures.

\begin{tabular}{|c|c|c|c|c|c|c|c|c|}
\hline \multirow{2}{*}{\multicolumn{2}{|c|}{ EEG changes }} & \multicolumn{2}{|c|}{ Seizure (no. $=22$ ) } & \multicolumn{2}{|c|}{ No Seizure $($ no. $=28)$} & \multirow{2}{*}{ Test value } & \multirow{2}{*}{$\mathrm{P}$-value } & \multirow{2}{*}{ Sig. } \\
\hline & & No. & $\%$ & No. & $\%$ & & & \\
\hline \multirow{2}{*}{ EEG } & Normal & 4 & $18.2 \%$ & 18 & 64.3 & \multirow{2}{*}{4.539} & \multirow{2}{*}{0.033} & \multirow{2}{*}{$S$} \\
\hline & Abnormal & 18 & $81.8 \%$ & 10 & $35.7 \%$ & & & \\
\hline \multirow{2}{*}{ Background } & Low voltage & 9 & $40.9 \%$ & 4 & $14.3 \%$ & \multirow{2}{*}{4.539} & \multirow{2}{*}{0.033} & \multirow{2}{*}{$S$} \\
\hline & Normal & 13 & $59.1 \%$ & 24 & $85.7 \%$ & & & \\
\hline \multirow{4}{*}{ Discharges } & No & 4 & $18.2 \%$ & 18 & $64.3 \%$ & \multirow{4}{*}{11.777} & \multirow{4}{*}{0.008} & \multirow{4}{*}{ HS } \\
\hline & Polyspikes & 2 & $9.1 \%$ & 0 & $0.0 \%$ & & & \\
\hline & Sharp waves & 7 & $31.8 \%$ & 4 & $14.3 \%$ & & & \\
\hline & Spikes & 9 & $40.9 \%$ & 6 & $21.4 \%$ & & & \\
\hline \multirow{4}{*}{ Site } & Centroparital & 9 & $40.9 \%$ & 3 & $10.7 \%$ & \multirow{4}{*}{11.758} & \multirow{4}{*}{0.008} & \multirow{4}{*}{ HS } \\
\hline & Frontotempotal & 6 & $27.3 \%$ & 4 & $14.3 \%$ & & & \\
\hline & Multifocal & 3 & $13.6 \%$ & 3 & $10.7 \%$ & & & \\
\hline & No & 4 & $18.2 \%$ & 18 & $64.3 \%$ & & & \\
\hline \multirow{3}{*}{ Side } & LF & 9 & $40.9 \%$ & 4 & $14.3 \%$ & \multirow{3}{*}{10.869} & \multirow{3}{*}{0.004} & \multirow{3}{*}{ HS } \\
\hline & No & 4 & $18.2 \%$ & 18 & $64.3 \%$ & & & \\
\hline & RT & 9 & $40.9 \%$ & 6 & $21.4 \%$ & & & \\
\hline
\end{tabular}

P-value > 0.05: Non significant (NS); P-value < 0.05: Significant (S); P-value < 0.01: highly significant (HS). *: Chi-squaretest; $\bullet$ : Independent t-test. 


\section{Discussion}

In our studied neonates, 56\% had abnormal EEG. EEG abnormalities were as following (26\%) around one third of the cases had low voltage background indicator of neurodevelopmental complications later on, (30\%) had spikes that may be an indicator of long term complications as epilepsy, (22\%) had sharp waves. Regarding the site of EEG abnormalities (24\%) were Centro parietal and (20\%) frontotemporal. Regarding the side of EEG abnormality, (30\%) were right sided ands (26\%) were left sided.

A large number of previous studies have shown that EEG is a very good predictor of neurodevelopmental outcomes [9] [10] (Neubauer et al., 2011; Volpe, 2008), while a few studies suggested limited value in predicting outcome [11] [12] (Bourez-Swart et al., 2009; Spitzmiller et al., 2007). Severely abnormal EEG background activity was associated with severe injury and neurodevelopmental outcome, while a mild-to-moderate impairment in EEG background was not a significant prognostic value [13] [14] (Song et al., 2015; Evans et al., 2010).

Zhang et al. (2013) [15] data revealed that the neonatal aEEG features of background pattern, cyclicity, and seizure activity were significantly associated with the neurodevelopmental outcomes at the age of one year. This result was largely consistent with previous EEG studies, which reported that EEG features, especially background EEG pattern, correlate well with neurological outcome in neonates with seizures [16] [17] (Pisani et al., 2012; Garfinkle and Shevell, 2011).

Also, many researchers found that interictal EEG activity was associated with the subsequent neurologic outcome of encephalopathic neonates accompanied by seizures [18] (Vasiljević et al., 2012). However, very few studies have focused on neonates with seizures and systematically investigated the predictive value of aEEG features for neurodevelopmental outcomes. The most relevant study we've found so far was undertaken by van der Heide et al. (2012) [19] which showed that the aEEG background pattern was significantly associated with subsequent neurologic outcome in neonates treated with two or more antiepileptic drugs. A clinical diagnosis of seizures is a challenging issue. One study revealed that $80 \%$ of electroencephalography (EEG) documented seizures were not accompanied by observable clinical seizures. EEGs are considered essential for the diagnosis of seizures in neonates [20] (Jensen, 2009).

Neonatal EEG demonstrates age-specific characteristics (frequencies and maturation patterns) so to provide an accurate interpretation of the neonatal EEG, it is important to know the conceptional age (CA) of Shany and Berger, (2011) [21].

Subtle convulsion is the most common type of neonatal seizures. About 35\% (one third) of neonates who didn't develop clinical seizures had abnormal EEG suggesting poor correlation between clinical and electroencephalographic detection of seizures. Background EEG abnormalities were detected in $26 \%$ of neonates with sepsis.

Conventional video-EEG monitoring is required to diagnose seizures accu- 
rately in neonates. This tool is resource-intense and has limited availability in many centers. While both clinical and EEG background features were predictive of neonatal seizures, the combination improved overall prediction of seizure occurrence and prediction of time to first seizure as compared with prediction models based solely on clinical or EEG features alone [22] (McCoy and Hahn, 2013).

The incidence of seizures is higher in the neonatal period than at any other time in life. Seizures and abnormalities of EEG background are associated with significant risk of mortality and long-term neurodevelopmental morbidities. In the neonatal population the majority of seizures are not clinically evident and go undetected without EEG monitoring [23] (Sansevere et al., 2019).

Ter Horst et al. (2010) [24] reported that severely abnormal EEG background patterns are not uncommon in infants with neonatal sepsis. Low voltage patterns within the first $48 \mathrm{~h}$ after admission are predictive of a poor neurological outcome or death. Studies that used conventional EEG in infants with bacterial meningitis reported similar findings [25] (Klinger et al., 2001).

Sepsis-associated brain dysfunction is mainly occurred in related to infections located outside the central nervous system that may develop before signs of other organ dysfunction appear with incidence ranging from $9 \%$ to $71 \%$ and the main clinical manifestations are seizures. Decreased brain perfusion is considered as a major determinant in the pathogenesis of brain dysfunction in sepsis, but various other mechanisms can be involved but vary from patient to patient. These mechanisms include inflammatory and non-inflammatory processes affecting the endothelial cells, glial cells and neurons resulting in blood-brain barrier breakdown, and derangements of intracellular metabolism, which can lead to cell death. Furthermore, systemic consequences of sepsis, such as prolonged inflammation, severe hypoxemia, and persistent hyperglycemia may contribute to aggravation of sepsis-induced brain dysfunction or injury. Morbidity and mortality are directly related to its severity. Diagnostic tools useful for detection of brain dysfunction are the electroencephalogram, brain MRI and somatosensory evoked potentials, but blood tests, such as plasma S-100b protein and neuron-specific enolase levels can also be helpful. EEG is almost sensitive but not specific for Sepsis induced brain dysfunction.

Ter Horst et al. (2010) [24] found that clinical seizures occurred frequently in more than $50 \%$ of infants with neonatal sepsis. The high incidence of clinical seizures might be explained by the fact that all these infants were admitted to a level III NICU and are therefore proportionally the most severely ill group of infants suffering from neonatal sepsis and/or meningitis. In $60 \%$ of the infants with clinical seizures, there were no abnormalities visible on EEG. It is known that brief seizures may not by EEG [26] (Toet et al., 2002). It can also be a reflection of the successful treatment of seizures because a majority of these infants were already treated with AED before EEG was recorded. Another explanation might be that diagnosing clinical seizures remains very difficult; a study using 
continuous video-EEG monitoring showed that only $27 \%$ of suspected clinical seizures had corresponding electrographic manifestations [27] (Murray et al., 2008).

We found poor correlation between clinical and EEG detection of seizures on comparing the EEG changes between neonates with and without clinical Seizures, in neonates with clinical seizures $18.2 \%$ had no EEG abnormalities and 81.2\% had abnormal EEG. In neonates with NO clinical seizures, $35.7 \%$ had EEG abnormalities and $64.3 \%$ had normal EEG.

(Wirrell EC) [28] The value of EEG in the diagnosis and classification of epilepsy is well-accepted. However, there are conflicting results from studies on the use of EEG as a predictor of prognosis in genetic generalized epilepsy (GGE).

\section{Conclusion}

EEG may be a useful bedside tool to assess neurological complication of neonatal sepsis. EEG monitoring should be incorporated in the work up for neonatal sepsis to detect sepsis-related neurological complications. High risk neonates including other than asphyxia including neonatal sepsis should be assessed using both clinical and EEG to detect neonatal seizures. Long term follows up for neonates with EEG abnormalities for early identification and treatment of neurodevelopmental abnormalities.

\section{Recommendations}

- High risk neonates including those with sepsis should be assessed using both clinical and EEG to detect neonatal seizures.

- EEG monitoring should be incorporated in the work up for neonatal sepsis to detect sepsis-related neurological complications.

- Long term follows up for neonates with EEG abnormalities for early identification and treatment of neurodevelopmental abnormalities.

\section{Conflicts of Interest}

The authors declare no conflicts of interest regarding the publication of this paper.

\section{References}

[1] Yismaw, A.E., Abebil, T.Y., Biweta, M.A. and Araya, B.M. (2019) Proportion of Neonatal Sepsis and Determinant Factors among Neonates Admitted in University of Gondar Comprehensive Specialized Hospital Neonatal Intensive Care Unit Northwest Ethiopia 2017. BMC Research Notes, 12, Article No. 542. https://doi.org/10.1186/s13104-019-4587-3

[2] Katie, M.S. (2019) A Comparative Review of Equine SIRS, Sepsis, and Neutrophils. Frontiers in Veterinary Science, 6, 69.

[3] Paczosa, M.K. and Mecsas, J. (2016) Klebsiella pneumoniae: Going on the Offense with a Strong Defense. Microbiology and Molecular Biology Reviews, 80, 629-661. https://doi.org/10.1128/MMBR.00078-15 
[4] Molnar, L., Fülesdi, B., Németh, N. and Molnár, C. (2018) Sepsis-Associated Encephalopathy: A Review of Literature. Neurology India, 66, 352-361. https://doi.org/10.4103/0028-3886.227299

[5] Gilmore, E.J., Gaspard, N., Choi, H.A., Cohen, E., Burkart, K.M., Chong, D.H., Claassen, J. and Hirsch, L.J. (2015) Acute Brain Failure in Severe Sepsis: A Prospective Study in the Medical Intensive Care Unit Utilizing Continuous EEG Monitoring. Intensive Care Medicine, 41, 686-694. https://doi.org/10.1007/s00134-015-3709-1

[6] Talebian, A., Jahangiri, M., Rabiee, M., et al. (2015) The Etiology and Clinical Evaluations of Neonatal Seizures in Kashan, IRAN. Iranian Journal of Child Neurology, 9, 29-35.

[7] Grova, N., Schroeder, H., Olivier, J.L. and Turner, J.D. (2019) Epigenetic and Neurological Impairments Associated with Early Life Exposure to Persistent Organic Pollutants. International Journal of Genomics, 2019, Article ID: 2085496. https://doi.org/10.1155/2019/2085496

[8] Ramantani, G., Maillard, L. and Koessler, L. (2016) Correlation of Invasive EEG and Scalp EEG. Seizure, 41, 196-200. https://doi.org/10.1016/j.seizure.2016.05.018

[9] Neubauer, O.D., Paro-Panjan, S.A. and Derganc, M. (2011) Recording Conventional and Amplitude-Integrated EEG in Neonatal Intensive Care Unit. European Journal of Paediatric Neurology, 15, 405-416. https://doi.org/10.1016/j.ejpn.2011.03.001

[10] Volpe, J.J. (2008) Neurology of the Newborn. 5th Edition, Saunders, Philadelphia, 203-237. https://doi.org/10.1016/B978-1-4160-3995-2.10005-6

[11] Bourez-Swart, M., Van Rooij, L., Rizzo, C., et al. (2009) Detection of Subclinical Electroencephalographic Seizure Patterns with Multichannel Amplitude-Integrated EEG in Full-Term Neonates. Clinical Neurophysiology, 120, 1916-1922. https://doi.org/10.1016/j.clinph.2009.08.015

[12] Spitzmiller, R.E., Phillips, T., Meinzen-Derr, J. and Hoath, S.B. (2007) Amplitude-Integrated EEG Is Useful in Predicting Neurodevelopmental Outcome in Full-Term Infants with Hypoxic-Ischemic Encephalopathy: A Meta-Analysis. Journal of Child Neurology, 22, 1069-1078. https://doi.org/10.1177/0883073807306258

[13] Song, J., Xu, F.L., Wang, L.S., et al. (2015) Early Amplitude-Integrated Electroencephalography Predicts Brain Injury and Neurological Outcome in Very Preterm Infants. Scientific Reports, 5, Article No. 13810. https://doi.org/10.1038/srep13810

[14] Evans, E., Koh, S., Lerner, J. and Garg, M. (2010) Accuracy of Amplitude Integrated EEG in a Neonatal Cohort. ADC Fetal \& Neonatal Edition, 95, F169-73. https://doi.org/10.1136/adc.2009.165969

[15] Zhang, D.D., Ding, H.Y., Liu, L., Hou, X.L., Sun, G.Y., Li, L., Liu, Y.Z., Zhou, C., $\mathrm{Gu}$, R.L. and Luo, Y.J. (2013) The Prognostic Value of Amplitude-Integrated EEG in Full-Term Neonates with Seizures. PLoS ONE, 8, e78960. https://doi.org/10.1371/journal.pone.0078960

[16] Pisani, F., Piccolo, B., Cantalupo, G., et al. (2012) Neonatal Seizures and Postneonatal Epilepsy: A 7-Y Follow-Up Study. Pediatric Research, 72, 186-193. https://doi.org/10.1038/pr.2012.66

[17] Garfinkle, J. and Shevell, M.I. (2011) Prognostic Factors and Development of a Scoring System for Outcome of Neonatal Seizures in Term Infants. European Journal of Paediatric Neurology, 15, 222-229. https://doi.org/10.1016/j.ejpn.2010.11.002

[18] Vasiljević, B., Maglajlić-Djukić, S. and Gojnić, M. (2012) The Prognostic Value of Amplitude-Integrated Electroencephalography in Neonates with Hypoxic-Ischemic 
Encephalopathy. Vojnosanitetski Pregled, 69, 492-499. https://doi.org/10.2298/VSP1206492V

[19] Van der Heide, M.J., Roze, E., Van der Veere, C.N., Ter Horsta H.J., Brouwer, O.F. and Bos, A.F. (2012) Long-Term Neurological Outcome of Term-Born Children Treated with Two or More Anti-Epileptic Drugs during the Neonatal Period. Early Human Development, 88, 33-38. https://doi.org/10.1016/j.earlhumdev.2011.06.012

[20] Jensen, F.E. (2009) Developmental Factors in the Pathogenesis of Neonatal Seizures. Journal of Pediatric Neurology, 7, 5-12.

[21] Shany, E. and Berger, I. (2011) Neonatal Electroencephalography: Review of a Practical Approach. Journal of Child Neurology, 26, 341-355.

https://doi.org/10.1177/0883073810384866

[22] Mccoy, B. and Hahn, C.D. (2013) Continuous EEG Monitoring in the Neonatal Intensive Care Unit. Journal of Clinical Neurophysiology, 30, 106-114. https://doi.org/10.1097/WNP.0b013e3182872919

[23] Sansevere, A.J., Kapur, K., Peters, J.M., et al. (2019) Seizure Prediction Models in the Neonatal Intensive Care Unit. Journal of Clinical Neurophysiology, 36, 186-194. https://doi.org/10.1097/WNP.0000000000000574

[24] Ter Horst, H.J., Van Olffen, M., Remmelts, H.J., De Vries, H. and Bos, A.F. (2010) The Prognostic Value of Amplitude Integrated EEG in Neonatal Sepsis and/or Meningitis. Acta Paediatrica, 99, 194-200. https://doi.org/10.1111/j.1651-2227.2009.01567.x

[25] Klinger, G., Chin, K., Otsubo, H., et al. (2001) Prognostic Value of EEG in Neonatal Bacterial Meningitis. Pediatric Neurology, 24, 28-31. https://doi.org/10.1016/S0887-8994(00)00221-6

[26] Toet, M.C., Van der Meij, W., De Vries, L.S., et al. (2002) Comparison between Simultaneously Recorded Amplitude Integrated Electroencephalogram (Cerebral Function Monitor) and Standard Electroencephalogram in Neonates. Pediatrics, 109, 772-779. https://doi.org/10.1542/peds.109.5.772

[27] Murray, D.M., Boylan, G.B., Ali, I., Ryan, C.A., Murphy, B.P. and Connolly, S. (2008) Defining the Gap between Electrographic Seizure Burden, Clinical Expression and Staff Recognition of Neonatal Seizures. ADC Fetal \& Neonatal Edition, 93, F187-91. https://doi.org/10.1136/adc.2005.086314

[28] Wirrell, E.C., Camfield, C.S., Camfield, P.R., Gordon, K.E. and Dooley, J.M. (2012) Long-Term Prognosis of Typical Childhood Absence Epilepsy Remission or Progression to Juvenile Myoclonic Epilepsy. Neurology, 47, 912-918.

https://doi.org/10.1212/WNL.47.4.912 